\title{
Developing native plant germplasm for national forests and grasslands in the Pacific Northwest-corrected table 1
}

\author{
I Vicky J Erickson
}

\section{ABSTRACT}

As originally published (Erickson 2008), Table 1 on pages 260 and 261 inadvertently had erroneous numbers in the "average seeds per kg" column.

\section{KEY WORDS}

grassland restoration, prairie, herbicide, restoration methods, seed addition, native diversity, invasive species

Erickson V. 2008. Developing native plant germplasm for national forests and grasslands in the Pacific Northwest. Native Plants Journal 9(3):255-266.

\section{AUTHOR INFORMATION}

Vicky J Erickson

Regional Geneticist

USDA Forest Service,

Pacific Northwest Region

2517 SW Hailey Avenue

Pendleton, OR 97801

verickson@fs.fed.us

\section{TABLE 1}

Quantity $(\mathrm{kg})$ of cleaned seeds needed to establish a 1-ha production field and estimated first- and second-yields in the Columbia Basin of eastern Oregon and Washington.

\begin{tabular}{|c|c|c|c|c|c|}
\hline Species & $\begin{array}{l}\text { Sowing rates } \\
\text { (kg of clean seeds } \\
\text { to sow per ha) }\end{array}$ & $\begin{array}{l}\text { Average first-y } \\
\text { yields (kg/ha)y }\end{array}$ & $\begin{array}{l}\text { Average second-y } \\
\text { yields }(\mathrm{kg} / \mathrm{ha})^{y}\end{array}$ & $\begin{array}{l}\text { Average } \\
\text { seeds per kg }\end{array}$ & $\begin{array}{l}\text { Average } \\
\text { germination/ } \\
\text { purity of } \\
\text { harvested } \\
\text { seeds (\%) }\end{array}$ \\
\hline $\begin{array}{l}\text { Bluebunch wheatgrass } \\
\text { (Pseudoroegneria spicata } \\
\text { (Pursh) A. Löve) }\end{array}$ & 9 & 225 & 340 & 308600 & $75 / 95$ \\
\hline $\begin{array}{l}\text { Blue wildrye } \\
\text { (Elymus glaucus Buckley) }\end{array}$ & 7 & 500 & 225 & 242500 & $65 / 96$ \\
\hline $\begin{array}{l}\text { Bottlebrush squirreltail } \\
\text { (Elymus elymoides (Raf.) } \\
\text { Swezey) or Big squirreltail } \\
\text { (Elymus multisetus M.E. Jones) }\end{array}$ & 7 & 0 & 140 & 242500 & $75 / 90$ \\
\hline
\end{tabular}

\begin{tabular}{lccccc}
$\begin{array}{l}\text { California oatgrass } \\
\text { (Danthonia californica Bol.) }\end{array}$ & 9 & 30 & 280 & 275600 & $75 / 90$ \\
\hline Great Basin wildrye & 9 & 30 & 180 & 286600 & $75 / 95$
\end{tabular}

(Leymus cinereus

(Scribn. \& Merr.) A. Löve)

\begin{tabular}{|c|c|c|c|c|c|}
\hline $\begin{array}{l}\text { Idaho fescue } \\
\text { (Festuca idahoensis Elmer) }\end{array}$ & 5 & 60 & 450 & 992100 & $75 / 90$ \\
\hline $\begin{array}{l}\text { Indian ricegrass } \\
\text { Oryzopsis hymenoides (Roem. } \\
\text { \& Schult.) Ricker ex Piper }\end{array}$ & 7 & 0 & 225 & 264600 & $80 / 85$ \\
\hline $\begin{array}{l}\text { Lemmon's needlegrass } \\
\text { (Achnatherum lemmonii } \\
\text { (Vasey) Barkworth }\end{array}$ & 9 & 170 & 850 & 330700 & $50 / 95$ \\
\hline
\end{tabular}


Species

\section{Sowing rates Average first-y ( $\mathrm{kg}$ of clean seeds yields ( $\mathrm{kg} / \mathrm{ha})^{y}$ to sow per ha) ${ }^{\mathrm{z}}$}

\section{Average second-y} yields (kg/ha)y
Average seeds per $\mathrm{kg}$
Average germination/ purity of harvested seeds (\%)

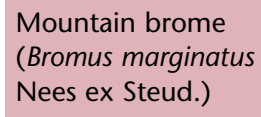

Needle and thread grass

(Hesperostipa comata

(Trin. \& Rupr.) Barkworth)

\section{Pinegrass}

(Calamagrostis

rubescens Buckley)

\section{Prairie Junegrass}

(Koeleria macrantha

(Ledeb.) Schult.)
11

7

2

2
900

0
680

170
154300

$85 / 90$

Sandberg's bluegrass

(Poa secunda J. Presl)

Slender hairgrass

(Deschampsia elongata

(Hook.) Munro)

Thurber's needlegrass

6

0

350

680

2896900

$75 / 92$

(Achnatherum thurberianum

(Piper) Barkworth)

\section{Tufted hairgrass}

3

0

150

5511600

$75 / 75$

Deschampsia cespitosa

(L.) P. Beauv)

Western needlegrass

6

$3 \quad 680$

400

4409200

$80 / 95$

(Achnatherum occidentale

(Thurb.) Barkworth)

\section{FAMILY ASTERACEAE}

\begin{tabular}{|c|c|c|c|c|c|}
\hline $\begin{array}{l}\text { Common yarrow } \\
\text { (Achillea millefolium L.) }\end{array}$ & 2 & 190 & 190 & 6613900 & $85 / 98$ \\
\hline $\begin{array}{l}\text { Pearly everlasting } \\
\text { (Anaphalis margaritacea } \\
\text { (L.) Benth.) }\end{array}$ & 1 & 60 & 60 & 17637000 & $60 / 85$ \\
\hline
\end{tabular}

Source: PNW Forest Service seed production database.

$\mathrm{Z}$ Sowing rate will need to be increased if germination and/or purity of seeds are lower than recommended values.

$\mathrm{y}$ Yield figures assume a late summer or fall sowing in year 0 .

Conversions: $\mathrm{kg} / \mathrm{ha} \times 0.89=\mathrm{lb} / \mathrm{ac}$; seeds $/ \mathrm{kg} \times 0.45=$ seeds $/ \mathrm{lb}$ 